\section{Localizing the EGF receptor}

To the editor - The epidermal growth factor receptor (EGFR) is cotranslationally inserted into the endoplasmic reticulum (ER) membrane as a type I transmembrane protein. This is a highly regulated process involving inherent topogenic and anchoring sequences. Furthermore, the lumenal/extracellular part of the receptor is subsequently glycosylated and folded, and the extracellular domain eventually possesses several stabilising disulphide bonds. The transmembrane part of the receptor is very hydrophobic. In mutants of the insulin receptor, where one or more hydrophobic residues of the transmembrane segment were instead polar, the entire protein was transported into the ER lumen and eventually secreted. An event whereby the full-length EGFR after synthesis and membrane insertion could be translocated out of the membrane, into the cytoplasm and subsequently into the nucleus by way of a nuclear localization sequence, as proposed by Lin et al. ${ }^{1}$, would involve yet unresolved and exciting mechanisms. But no such mechanism is substantiated, nor suggested, in the paper published in the recent issue of Nature Cell Biology $y^{1}$.

We strongly doubt the interpretation of the authors' results. It is important to realise that the ER membrane is contiguous with the nuclear membrane. Given this fact, proteins localizing to the ER could easily be misinterpreted as nuclear. This could explain the observation of the EGFR localizing to nuclei. The immunofluorescence data provided do not convincingly demonstrate nuclear EGFR localization. It is difficult to distinguish between the nuclei and the cytosol, and the optical section could be too thick to give the required resolution. Furthermore, the purity of nuclear fractions is highly critical with respect to interpretations regarding transport of radiolabelled EGFR to the nucleus.

It is interesting that a new track from the plasma membrane to the ER was recently described ${ }^{2}$. Potentially, similar mechanisms for EGFR intracellular routing could be involved.

\section{Oksvold, H. Huitfeldt, E. Stang and I. Madshus*}

University of Oslo, Department of Pathology, Oslo, Norway 0027

*e-mail:ghp@i.h.madshus@labmed.uio.no
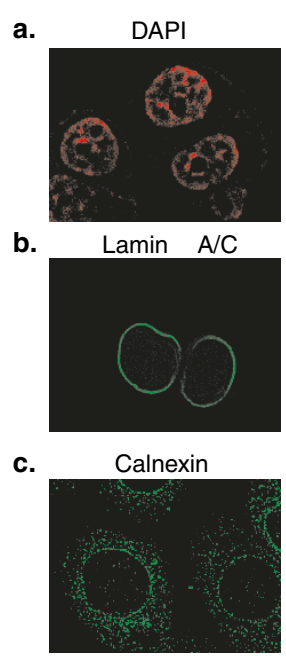

d.

Calregulin

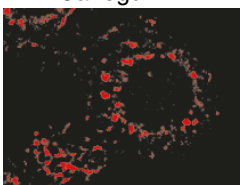

EGFR

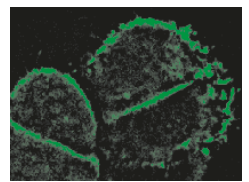

EGFR

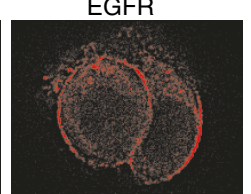

EGFR

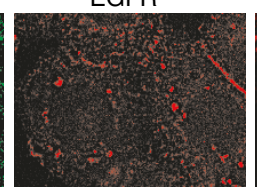

EGFR

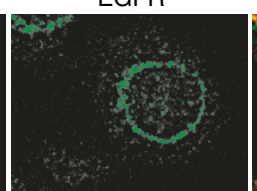

Merge

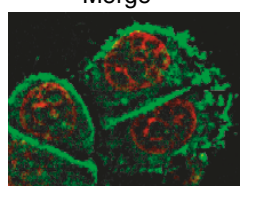

Merge

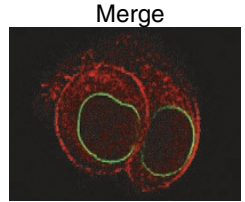

Merge

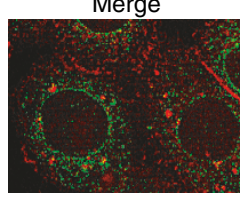

Merge

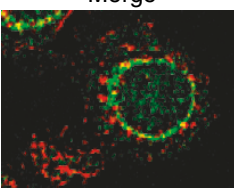

DAPI

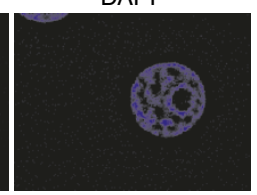

Figure 1. Double immunofluorescence staining and confocal analyses of nuclear EGFR and various organelle-specific markers in MDA-MB-468 cells. a, EGFR staining using rabbit anti-EGFR antibody followed by FITC-labeled goat anti-rabbit IgG. b. Nuclear lamin staining using mouse anti-lamin A/C antibody followed by Texas-Red-labeled goat anti-mouse IgG. c, An overlay image of $\mathbf{a}$ and $\mathbf{b}$. d, EGFR staining using rabbit anti-EGFR antibody followed by FITC-labeled goat anti-rabbit IgG. e, Bip/GRP78 staining of endoplasmic reticulum using mouse anti-Bip/GRP78 followed by Texas-Red-labeled goat anti-mouse IgG. f, An overlay image of $\mathbf{d}$ and $\mathbf{e}$. $\mathbf{g}$, EGFR staining using rabbit anti-EGFR antibody followed by FITC-labeled goat anti-rabbit IgG. $\mathbf{h}$, Calnexin staining of endoplasmic reticulum using mouse anti-Calnexin followed by Texas-Red-labeled goat anti-mouse IgG. i, An overlay image of $\mathbf{g}$ and $\mathbf{h}$. Nuclear EGFR is clearly colocalized with lamin in the nucleus (a-c). The arrowheads mark location of nucleus. No significant co-localization between EGFR and two endoplasmic reticulum markers [e.g. Bip/GRP78 (d-f) or Calnexin (g-i)] in the nucleus was observed.

Reply - Indeed, we agree that EGFR nuclear localization will involve yet unresolved and exciting mechanisms as pointed out by Madshus and co-workers. As mentioned in the discussion section of our manuscript: "It is not clear how EGFR is translocated to the nucleus...." We did discuss the possibility that the nuclear pore complex (the Ran-Importin pathway) might be involved. The mechanism suggested by Madshus and co-workers stems from "the mutants of insulin receptor, where one or more hydrophobic residues of the transmembrane segment were instead polar." But no such mutation has been reported in EGFR, and no evidence supports that mutation is required for nuclear localization of EGFR in our Nature Cell Biology paper $^{1}$. Thus, without further investigation, it is premature to propose the same type of mechanism. It should be pointed out that nuclear translocation of fibroblast growth factor receptor 1 has recently been shown to occur via an importin- $\beta$-dependent mechanism $^{3}$ and may be involved in transcriptional activity ${ }^{4}$. Also, mutations in the transmembrane segment of HER-2/neu, a member of EGFR family that changes a hydrophobic valine residue to a hydrophilic glutamic acid have been reported. The mutation enhances transforming activity of the HER-2/neu gene and the mutant is considered as a transforming oncogene $e^{5}$. It is not yet known whether the Val $\rightarrow$ Glu mutation could be involved in nuclear localization.

The major contribution of our paper ${ }^{4}$ is to demonstrate a new function for the EGFR - transcriptional activity - and to reinforce the significance of nuclear localization of the EGFR (and other receptor tyrosine kinases such as receptors for insulin, nerve growth factor and others, as mentioned in the discussion of the paper) that have been overlooked for decades. It is certainly important to determine the detailed mechanism of the EGFR nuclear translocation. But any mechanisms should be supported by solid experimental evidence.

Regarding the interpretation of our results, we have evidence using confocal microscopy (Fig. 1) and 2D-deconvolution (Fig. 2) analyses that nuclear EGFR does not co-localize with ER markers such as Calnexin and Bip/GRP78. Furthermore, the nuclear EGFR is colocalized with nuclear lamin, supporting the idea that EGFR is in the nucleus. In addition, our chromatin-IP experiment clearly demonstrated that 

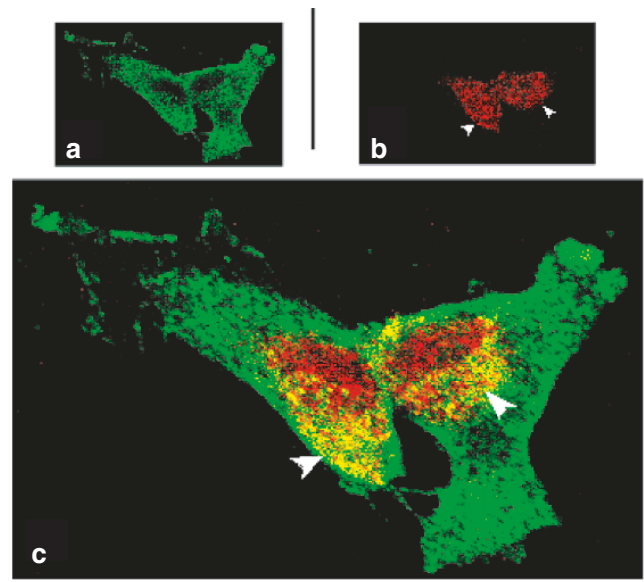
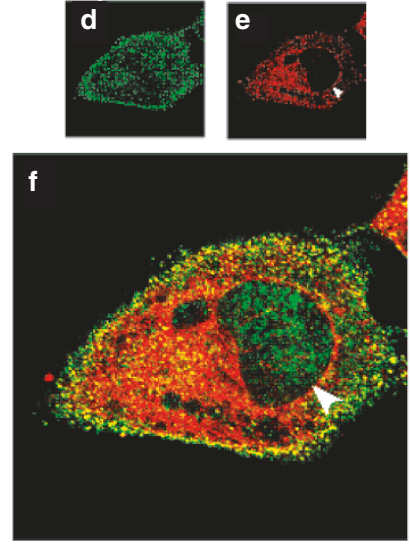
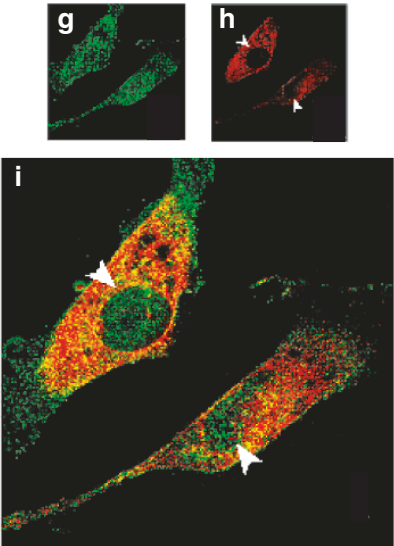

Figure 2. Localization of EGFR in the nucleus and other organelles in MDA-MB-468 cells. a, EGFR staining was performed using mouse anti-EGFR antibody (Ab-12, NeoMarkers) followed by FITC-labeled donkey anti-mouse IgG (Jackson ImmunoResearch). EGFR was found in the nucleus as well as in the cytoplasm. b. To confirm the nuclear localization of EGFR, the nuclei were stained using mouse anti-lamin A/C antibody (Santa Cruz Biotechnology) followed by FITC-labeled donkey anti-mouse IgG. EGFR staining using rabbit anti-EGFR antibody (sc03, Santa Cruz Biotechnology) followed by Texas-Red-labeled goat anti-rabbit IgG (Jackson ImmunoResearch). The overlay image of the two shows that EGFR signals are also inside the areas confined by lamin staining, i.e., the nuclei. c, ER staining using mouse anti-Calnexin antibody (BD Transduction Laboratories) followed by FITC-labeled donkey anti-mouse IgG. EGFR staining using rabbit anti-EGFR antibody followed by Texas-Red-labeled goat anti-rabbit IgG. d, ER staining using rabbit anti-Calregulin antibody (Santa Cruz Biotechnology) followed by Texas-Red-labeled goat anti-rabbit IgG. EGFR staining using mouse anti-EGFR antibody followed by FITC-labeled donkey anti-mouse IgG. Nuclear staining using DAPI further confirms the nuclear area defined by ER staining. $\mathbf{a}$ and $\mathbf{b}$ show nuclear localization of EGFR; and $\mathbf{c}$ and $\mathbf{d}$ clearly demonstrate that most of nuclear EGFR staining is not co-localized with ER staining. Similar results were obtained when another cell line, A431 was used for staining (data not shown).

EGFR can bind directly to the promoter region of cyclin D1 in vivo (Fig. 7b in the paper). The protein localized in ER membrane will not associate with chromatin; therefore, direct binding of EGFR to a promoter in vivo is rock-solid data to support the nuclear localizaton of EGFR. As to the confocal results shown in Fig. 1d (ref. 4), the picture might be too small to have good resolution. The original figure has much better resolution. But all the biochemical evidence (Figs $2-7$ in ref. 4 ) and the data presented in this correspondence clearly supports the localization of EGFR in the nucleus.
1. Lin, S.-Y., et al. Nature Cell Biol. 3, 802-808 (2001). 2. Pelkmans, L., Kartenbeck, J, \& Helenius, A. Nature Cell Biol. 3, 473-483 (2001)

3. Reilly, J. F. \& Maher, P. A. J. Cell Biol. 152, 1307-1312 (2001). 4. Peng, H. et al. Mol. Biol. Cell 12, 449-462 (2001).

5. Bargmann, C. I., Hung, M. C. \& Weinberg, R. A. Cell 45, 649-657 (1986)

Lilly Bourguignon*, Keng-hsueh Lan†, Patrick Singleton*, Shiaw-Yih Lin§, Dihua Yu†§, and Mien-Chie Hung†‡§

${ }^{*}$ Department of Medicine, University of California San Francisco and Endocrine Unit at the VA Medical Center, San Francisco, CA 94121, USA;
†Department of Surgical Oncology and ¥Department of Molecular and Cellular Oncology, University of Texas MD Anderson Cancer Center, Houston, TX 77030-4009, USA Se-mail:mhung@mdanderson.org

\section{Cells and gels}

To the editor - I read Michael Klymkowsky's recent review of my book, Cells, Gels and the Engines of Life (Nature Cell Biol. 3, E213; 2001), and find myself struggling to understand how his observations lead to his conclusions.

Klymkowsky admits that at least some of the challenges to current orthodoxy may be substantive. Yet, he dismisses such challenges as "anecdotal," notwithstanding some 450 cited references. He then goes on to declare that the "cross-checked" findings of many studies (none cited) leave little room for doubt that current views must be rock solid. Given such certainty, he is forced to conclude that questions such as those raised in the book do harm to science.

I was taught that scientific theories could never be proved; that no matter how much evidence could be marshalled in support of a theory, a new, conflicting observation has the potential to turn that theory on its ear. Klymkowsky has apparently learned from a different teacher. With a sweep of the hand, he dismisses the wealth of contradictory evidence presented in the book as "anecdotal," and prefers to bank on all of those ineffable "cross-checked" findings. Perhaps he could explain why findings that fit current views should be given more weight than those that do not fit.

The author also takes a jab at the presentation's "folksy" style. Deviating from the stuffy, jargon-filled style that typifies much scientific writing (and obscures flaws in reasoning) is regarded as unscientific.

Klymkowsky may be surprised to find that the material he dismisses as "muckmaking" is in growing demand worldwide-on the plenary agenda at international scientific venues, and increasingly in the classroom at major universities. Cells, Gels and the Engines of Life is becoming a scientific best-seller, with translations pending in three languages. The community is evidently hungry for a critical, no-holds barred, evaluation of entrenched paradigms, and for exposure to potentially more productive paradigms - even if the presentation style may border on the "folksy."

Gerald H. Pollack

Dept. of Bioengineering, University of Washington, Seattle, Washington 98195, USA e-mail:ghp@u.washington.edu 